\title{
Effect of sulphasalazine and its metabolites on the generation of reactive oxygen species
}

\author{
Y MIYACHI, A YOSHIOKA, S IMAMURA, AND Y NIWA \\ From the Department of Dermatology, Faculty of Medicine, Kyoto University, Kyoto and Niwa Institute for \\ Immunology, Kochi, Japan
}

SUMMARY The relative in vitro anti-oxidant efficacy of sulphasalazine (salicylazosulphapyridine, SASP) and its metabolites (5-aminosalicylic acid, 5-ASA; sulphapyridine, SP) was examined by studying their effects on the generation of reactive oxygen species (ROS) using zymosanstimulated polymorphonuclear leucocytes (PMNs) and a cell free, xanthine-xanthine oxidase system. Salicylazosulphapyridine, 5-ASA, and SP showed anti-oxidant effects to the various degrees. In particular, production of $\mathrm{OH}^{\bullet}$, which is one of the most potent reactive oxygen species, was remarkably suppressed by 5-ASA dose relatedly. These findings suggest that SASP and its metabolites play an important role in the inhibition of respiratory bursts. As the potent products of the respiratory burst by polymorphonuclear leucocytes are thought to be important inflammatory mediators, suppression of toxic reactive oxygen species generation by these agents may partly explain the therapeutic efficacy of SASP in ulcerative colitis, which is characterised by an acute mucosal inflammation dominated by polymorphonuclear leucocytes accumulation.

Sulphasalazine (SASP) has been shown to be useful in the therapy and prophylaxis of ulcerative colitis.' Nearly all the SASP taken orally reaches the colon intact, where it is converted by bacteria into sulphapyridine (SP) and 5-aminosalicylic acid (5-ASA). ${ }^{2}$ The active therapeutic moiety of SASP is 5-ASA and SP functions as a carrier ensuring that the 5-ASA is liberated within the colon. ${ }^{3} 5$-aminosalicylic acid may be less toxic and thus may be an alternative to SASP in inflammatory bowel diseases. ${ }^{4}$

The mode of action of SASP has been a matter of dispute. One attractive hypothesis is that prostaglandins are major mediators of inflammation in ulcerative colitis and that SASP reduces inflammation by blocking prostaglandin synthesis. ${ }^{5-7}$ Whether the cause of the inflammation first involves increased prostaglandin synthesis, however, or whether inflammation causes increase of prostaglandins ${ }^{8}$ is unknown. A major argument against this hypothesis is that other drugs that inhibit prostaglandin syn-

Address for correspondence: Dr Y Miyachi, Department of Dermatology, Faculty of Medicine, Kyoto University, Sakyo-ku, Kyoto 606, Japan.

Received for publication 16 June 1986 thesis are not useful in the treatment of ulcerative colitis. $^{-11}$ Ulcerative colitis is characterised by an acute mucosal inflammation dominated by polymorphonuclear leucocyte accumulation; it is possible that the effect of SASP is primarily on polymorphonuclear leucocyte activity, and that the reduction of prostaglandin levels after such therapy is secondary to the reduced inflammatory response.

Several consecutive steps are involved in the polymorphonuclear leucocyte activation. Inhibition of any or some of these steps would result in a reduced inflammatory response and tissue damage. The efficacy of SASP in the treatment of ulcerative colitis may relate to the inhibition of the synthesis of chemotactic compounds. ${ }^{12}$ It has also been reported that SASP and its metabolites inhibit different stages of polymorphonuclear leucocyte activation such as superoxide production. ${ }^{13}$ Several anti-inflammatory drugs including aspirin have been shown to reduce superoxide ${ }^{14}$ and hydroxyl radical levels. ${ }^{15}$

In the present study, we have investigated the effect of SASP and its metabolites on the generation of reactive oxygen species, as oxidants produced by polymorphonuclear leucocytes may play an important role in causing tissue damage. 


\section{Methods}

\section{MATERIALS}

PREPARATION OF POLYMORPHONUCLEAR LEUCOCYTES FOR REACTIVE OXYGEN SPECIES ASSAYS

Polymorphonuclear leucocytes were prepared from heparinised peripheral venous blood taken from healthy adult volunteers (age 21-38 years) on no treatment as previously described.$^{16}$ After centrifugation of the blood over a Ficoll-Hypaque gradient, the cell pellet, containing polymorphonuclear leucocytes and erythrocytes, was washed with a saline solution and resuspended in plasma containing dextran 170 (molecular weight, 170000) at a final concentration of $1 \%$. The polymorphonuclear leucocytes were recovered after sedimentation at unit gravity, and the few contaminating erythrocytes were lysed by treatment of the preparation with $0 \cdot 876 \% \mathrm{NH}_{4} \mathrm{Cl}$. For the assays of reactive oxygen species, the polymorphonuclear leucocytes were then resuspended in a KrebsRinger phosphate buffer containing $5 \mathrm{mM}$ glucose and $1 \mathrm{mg} / \mathrm{ml}$ gelatin. Gelatin was added to prevent the polymorphonuclear leucocytes from adhering to the test tubes. It was excluded, however, from the medium for $\mathrm{OH}^{*}$ generation assay because of its inhibitory effect.

REACTIVE OXYGEN SPECIES GENERATION ASSAY Fresh opsonised zymosan was prepared before each experiment by incubating $11 \mathrm{mg}$ zymosan (Sigma) with $1 \mathrm{ml}$ autologous serum at $37^{\circ} \mathrm{C}$ for 30 minutes. The $\mathrm{O}_{2}{ }^{-}$formation was determined according to the method of Johnston and Lehmeyer. ${ }^{17}$ Polymorphonuclear leucocytes $\left(4 \times 10^{6}\right)$ were preincubated for 10 minutes with $1 \mathrm{mg} / \mathrm{ml}$ opsonised zymosan. After the addition of $0 \cdot 1 \mathrm{mM}$ ferricytochrome c (Type III, Sigma), the cells were further incubated at $37^{\circ} \mathrm{C}$ for 30 minutes. Immediately after sedimentation of the polymorphonuclear leucocytes and opsonised zymosan by centrifugation, the supernatants were assayed for reduced cytochrome $\mathrm{c}$ by measuring the absorbance at $550 \mathrm{~nm}$ with a spectrophotometer (Hitachi Co, Tokyo, Japan). Superoxide dismutase was added to the polymorphonuclear leucocyte medium at a concentration of $400 \mathrm{U} / \mathrm{ml}$. Inhibition of the cytochrome $\mathrm{c}$ reduction was confirmed. Generation of $\mathrm{H}_{2} \mathrm{O}_{2}$ was determined by measuring the decrease in the fluorescence intensity of the scopoletin (Sigma) because of its peroxidase-mediated oxidation by $\mathrm{H}_{2} \mathrm{O}_{2} \cdot{ }^{18}$ After incubation of $2.5 \times 10^{6}$ polymorphonuclear leucocytes in Krebs-Ringer phosphate buffer with $1 \mathrm{mg} / \mathrm{ml}$ opsonised zymosan for 10 minutes at room temperature, $0.1 \mathrm{ml}$ of $50 \mathrm{mM}$ scopoletin in Krebs-Ringer phosphate buffer and $0 \cdot 1$ $\mathrm{ml}$ of $1 \mathrm{mg} / \mathrm{ml}$ horseradish peroxidase (Type II, Sigma) in phosphate-buffered saline were added. The rate of decrease in the fluorescence intensity of the scopoletin within 30 minutes was measured with a fluorescence spectrophotometer (Hitachi). Incubation of supernatants with an excess of catalase $(600 \mathrm{U} / \mathrm{ml})$ inhibited the fluorescence reduction. Catalase heated at $130^{\circ} \mathrm{C}$ for 30 minutes served as the control.

The $\mathrm{OH}^{\bullet}$ was measured by the amount of the ethylene gas formed from $\alpha$-ketomethiol butylic acid (KMB, Sigma) plus the $\mathrm{OH}^{\bullet}$ generated by polymorphonuclear leucocytes. ${ }^{19}$ The polymorphonuclear leucocytes $\left(2 \times 10^{6}\right)$ in $2 \mathrm{ml}$ Krebs-Ringer phosphate buffer were preincubated with $1 \mathrm{mM} \alpha$-ketomethiol butylic acid at $37^{\circ} \mathrm{C}$ for five minutes. After the addition of $1 \mathrm{mg} / \mathrm{ml}$ opsonised zymosan, the incubation was continued for a further 10 minutes. The amount of $\mathrm{OH}^{\bullet}$ gas formed was assayed at 30 minutes using a gaschromatograph (Hitachi). The simultaneous addition of $10 \mathrm{mM}$ benzoate into the polymorphonuclear leucocyte medium reduced the peak $\mathrm{OH}^{\bullet}$ generation by $75 \%$. Chemiluminescence was measured in a scintillation spectrometer (Packard, IL, USA), according to the method of Allen and Loose $^{20}$ with slight modification. The polymorphonuclear leucocytes $\left(5 \times 10^{6}\right)$ in $3 \mathrm{ml}$ colourless Hanks' solution containing gelatin were incubated at $37^{\circ} \mathrm{C}$ for 10 minutes with opsonised zymosan in the absence of luminol. Chemiluminescence was monitored on a spectrometer, which was operated in the out-of-coincidence summation mode. All procedures were undertaken in the dark. Incubation of the polymorphonuclear leucocytes plus opsonised zymosan with $2 \mathrm{mM}$ xanthine reduced the peak chemiluminescence by $70 \%$.

EFFECTS OF SASP AND ITS METABOLITES ON REACTIVE OXYGEN SPECIES GENERATION FROM POLYMORPHONUCLEAR LEUCOCYTES

Salicylazosulphapyridine and SP were dissolved in a solution of $50 \%$ dimethyl sulphoxide and $50 \%$ ethanol. 5-aminosalicylic acid was dissolved in phosphate buffered saline. The solution was added to the polymorphonuclear leucocyte suspended medium of each reactive oxygen species assay system to make the final concentrations of 1,5 , and $10 \mu \mathrm{g} / \mathrm{ml}$. The final concentration of dimethyl sulphoxide was $0.5 \%$ and that of ethanol was also $0 \cdot 5 \%$. The same volume of dimethyl sulphoxide/ethanol containing buffer was added to the control to determine its effect on reactive oxygen species production. The same volume of phosphate buffered saline was added to the control in experiments of 5-ASA. As the sensitivity of the polymorphonuclear leucocytes to the zymosan stimulation varies from donor to donor, the 
effects of the agents on reactive oxygen species generation were expressed as the percentage of the experiment controls. Experiments were repeated four times using polymorphonuclear leucocytes from different donors. The mean of each assay in triplicate was calculated and compared with the appropriate control.

EFFECTS OF SASP AND ITS METABOLITES ON REACTIVE OXYGEN SPECIES GENERATION IN A XANTHINE-XANTHINE OXIDASE SYSTEM:

In separate experiments, the effects of the agents on reactive oxygen species generation were also examined in a cell free, xanthine-xanthine oxidase system. Instead of adding polymorphonuclear leucocytes and opsonised zymosan, $0.1 \mathrm{ml}$ of $13.5 \mathrm{mg}$ hypoxanthine (Sigma) in $50 \mathrm{ml}$ physiological saline plus $0.05 \mathrm{ml}$ of $50 \mathrm{mM}$ ethylenediaminetetraacetate (EDTA) were diluted in $2 \mathrm{ml}$ of Krebs-Ringer phosphate buffer $(\mathrm{pH}=7 \cdot 2-7 \cdot 4)$. Then, the agents in the dimethyl sulphoxide/ethanol solution or phosphate buffered saline were added to the medium to make the final concentrations of 1,5 , and $10 \mu \mathrm{g} / \mathrm{ml}$. The same volume of the solution (dimethyl sulphoxide/ethanol or phosphate buffered saline) was added to the control. Thereafter, $0.1 \mathrm{unit} / \mathrm{ml}$ of dialysed xanthine oxidase (Sigma) was added to generate reactive oxygen species and each reactive oxygen species was determined as described above. As the same dose of hypoxanthine plus xanthine oxidase did not necessarily produce the same amount of reactive oxygen species, the effects of the agents on reactive oxygen species levels were expressed as the percentage of the experiment controls. Experiments were repeated three times in triplicate. The mean of the triplicate assays was calculated and compared with the appropriate control.

VIABILITY OF POLYMORPHONUCLEAR

LEUCOCYTES AFTER THE EXPERIMENTS:

The viability of polymorphonuclear leucocytes after the assays with the agents was measured by means of the trypan blue dye test. When over $20 \%$ of the polymorphonuclear leucocytes obtained after the assay were stained with trypan blue, they were considered to have been damaged during the assay.

\section{CHEMICALS}

Salicylazosulphapyridine was a gift from Green Cross Co, Osaka, Japan. 5-aminosalicylic acid and SP were purchased from Sigma Chemical Co, St Louis, MO, USA.

STATISTICAL ANALYSIS

Analysis of results was by the paired $t$ test.

\section{Results}

Figure 1 shows the effect of SASP and its metabolites on the generation of $\mathrm{O}_{2}{ }^{-}$. SP decreased $\mathrm{O}_{2}{ }^{-}$level dose-dependently in the polymorphonuclear leucocyte system and SASP reduced the $\mathrm{O}_{2}{ }^{-}$production in the cell free xanthine-xanthine oxidase system.

As shown in Figure 2, 5-ASA had inhibitory effects on $\mathrm{H}_{2} \mathrm{O}_{2}$ generation both by the polymorphonuclear leucocytes and in the cell free generation system of hypoxanthine plus xanthine oxidase. Salicylazosulphapyridine showed modest inhibition at the highest concentration in the xanthine-xanthine oxidase system. Sulphapyridine showed no effect on $\mathrm{H}_{2} \mathrm{O}_{2}$ generation.

5-aminosalicylic acid clearly inhibited $\mathrm{OH}^{\bullet}$ production in the polymorphonuclear leucocyte system dose-dependently (Fig. 3). Salicylazosulphapyridine and SP did not affect the $\mathrm{OH}^{\bullet}$ level. These agents had no suppressive effect in the cell free, xanthine-xanthine oxidase system.

Figure 4 shows the effect of the agents on chemiluminescence. 5-aminosalicyclic acid significantly reduced the light emission dose relatedly. No effect was observed by SASP or 5-ASA in the polymorphonuclear leucocyte system. As no significant chemiluminescence was measured in the xanthine-xanthine oxidase system, only the data obtained in the polymorphonuclear leucocyte system are shown in Figure 4.

All of the polymorphonuclear leucocytes tested in this study showed less than $7 \%$ of the cells stained with trypan blue.

\section{Discussion}

We have shown that SASP and its metabolites are anti-oxidants. Salicylazosulphapyridine and 5-ASA had inhibitory effects on $\mathrm{H}_{2} \mathrm{O}_{2}$ generation presumably because of their direct reactive oxygen species quenching activities. Of great interest is that 5-ASA markedly suppressed the $\mathrm{OH}^{\bullet}$ production by the stimulated polymorphonuclear leucocytes but not in the cell free, xanthine-xanthine oxidase system. $\mathrm{OH}^{*}$ is one of the most toxic oxidants capable of causing tissue damage. This indicates that the inhibitory effect is exerted by an effect on cell functions. 5ASA also significantly reduced chemiluminescence dose dependently. Sulphapyridine slightly inhibited $\mathrm{O}_{2}$ - generation in the polymorphonuclear leucocytes system. These findings suggest that both SASP and its components affect respiratory bursts but with somewhat different modes of action with resultant modulation of inflammatory reactions. In particular, 5-ASA seems to play a larger part in suppressing 


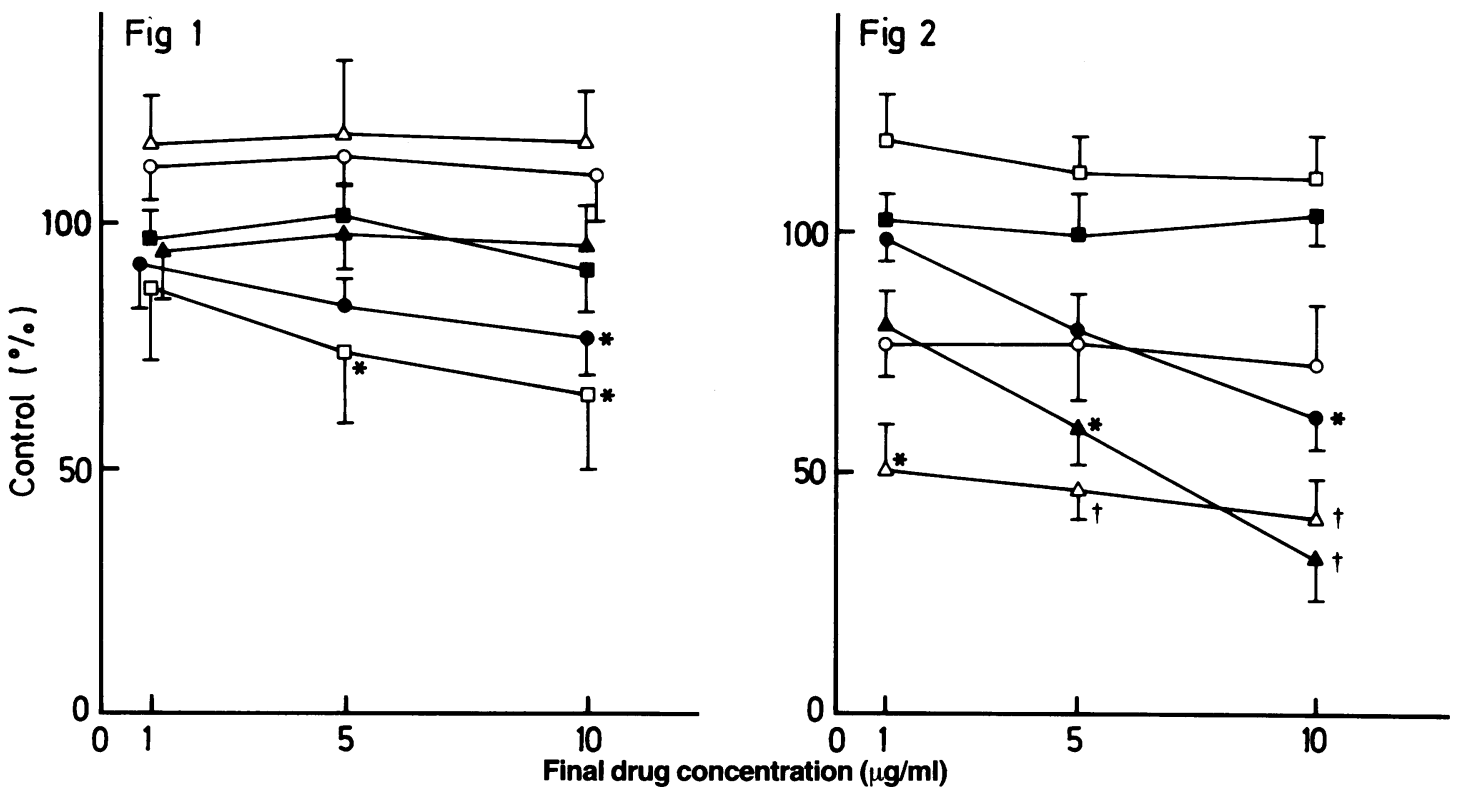

Fig. 1 Effects of SASP and its metabolites on superoxide $\left(\mathrm{O}_{2}^{-}\right)$generation. Agents $(1-10 \mu \mathrm{g} / \mathrm{ml})$ were examined both in a zymosan-stimulated polymorphonuclear leucocyte system (SASP: $\bigcirc-\bigcirc, 5-A S A: \triangle \longrightarrow \triangle, S P: \square-\square)$ and in a cell free, xanthine-xanthine oxidase system (SASP: - - 5-ASA: $\triangle-\triangle, S P: \square-\square)$. Results were expressed as the percentage of the controls. Each value denotes the mean $\pm S D$ of the assays and is compared with the appropriate control. ${ }^{*} p<0 \cdot 05$.

Fig. 2 Effects of SASP and its metabolites on hydrogen peroxide $\left(\mathrm{H}_{2} \mathrm{O}_{2}\right)$ generation. Agents $(1-10 \mu \mathrm{g} / \mathrm{ml})$ were examined both in a zymosan-stimulated polymorphonuclear leucocyte system (SASP: $\bigcirc-\bigcirc, 5-A S A: \triangle-\triangle, S P: \square-\square)$ and in a cell free, xanthine-xanthine oxidase system (SASP:O-O, 5-ASA: $\triangle-\Delta, S P: \square-\square)$. Results were expressed as the percentage of the controls. Each value denotes the mean $\pm S D$ of the assays and is compared with the appropriate control. ${ }^{*} p<0.05 ; \dagger p<0.01$.

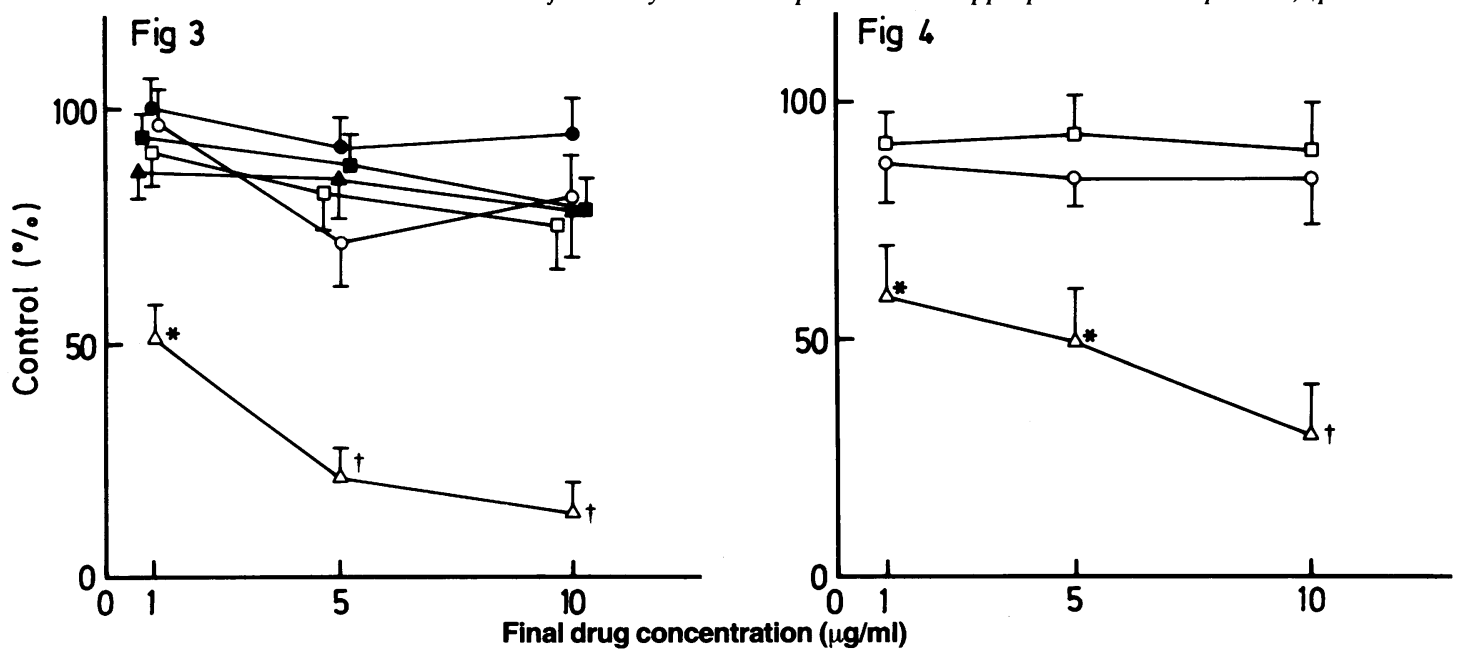

Fig. 3 Effects of SASP and its metabolites on hydroxyl radical $\left(\mathrm{OH}^{\bullet}\right)$ generation. Agents $(1-10 \mu \mathrm{g} / \mathrm{ml})$ were examined both in a zymosan-stimulated polymorphonuclear leucocyte system (SASP: $\bigcirc-O, 5-A S A: \triangle-\triangle, S P: \square-\square)$ and in a cell free, xanthine-xanthine oxidase system (SASP: ——, 5-ASA: $\triangle \longrightarrow \triangle, S P: \square-\square)$. Results were expressed as the percentage of the controls. Each value denotes the mean $\pm S D$ of the assays and is compared with the appropriate control. ${ }^{*} p<0 \cdot 05 ; \dagger p<0 \cdot 01$.

Fig. 4 Effects of SASP and its metabolites on chemiluminescence (CL). Agents $(1-10 \mu \mathrm{g} / \mathrm{ml})$ were examined in a zymosanstimulated polymorphonuclear leucocyte system (SASP: $\bigcirc-\bigcirc, 5-A S A: \triangle-\triangle, S P: \square-\square)$. In the cell free, xanthinexanthine oxidase system, no significant $C L$ was observed. Thus, the effects of the agents on chemiluminescence are not shown in the Figure. Results were expressed as the percentage of the controls. Each value denotes the mean $\pm S D$ of the assays and is compared with the appropriate control. ${ }^{*} p<0.05 ;+p<0.01$. 
polymorphonuclear leucocytes oxidative metabolism.

Molin and Stendah $l^{13}$ have reported that $0.5 \mathrm{mM}$ SASP reduces the $\mathrm{O}_{2}^{-}$production moderately and that 5-ASA shows no effect, whereas 0.5 and $0.1 \mathrm{mM}$ SP inhibit the $\mathrm{O}_{2}^{-}$activity significantly. Our experiments were designed to assess the effect of therapeutic serum concentrations of the drugs using lower doses, and thus could not detect the inhibitory effects of SASP on $\mathrm{O}_{2}{ }^{-}$generation, but confirmed those of SP in a dose-dependent fashion. Furthermore, we have extended their report on anti-oxidant effects of SASP and its metabolites by demonstrating their effects on other reactive oxygen species, in particular, on $\mathrm{OH}^{\bullet}, \mathrm{H}_{2} \mathrm{O}_{2}$ and chemiluminescence.

The role of reactive oxygen species produced by polymorphonuclear leucocytes in mediating inflammatory events has been studied recently. Reactive oxygen species generated by stimulated polymorphonuclear leucocytes can exert tissue injury at the site of inflammation. ${ }^{21}$ We have found that several inflammatory diseases are mediated in part by enhanced production of reactive oxygen species. ${ }^{22-27}$ Furthermore, some anti-inflammatory agents interfere with the polymorphonuclear leucocyte-derived reactive oxygen species generation, thus conferring protection against auto-oxidative tissue damage. ${ }^{2829}$ It has also been reported that superoxide radicals are involved in the pathogenesis of ischaemic mucosal lesions and that pretreatment with superoxide dismutase attenuated the necrosis of epithelium produced by three hours of ischaemia. ${ }^{31}$

Although SASP concentrations found in vivo: faecal water concentrations of 5-ASA are at least one hundred times more than those used in this study, ${ }^{31}$ corresponding serum concentrations were extremely low. ${ }^{32}$ If the accumulated polymorphonuclear leucocytes in colonic mucosa play a substantial part in the inflammatory process of ulcerative colitis, the polymorphonuclear leucocytes' activation should be affected by the agents at the concentrations found in colonic mucosa presumably comparable with those of serum but not in the stool. Salicylazosulphapyridine has been reported to be a potent inhibitor of prostaglandin degradation and $50 \%$ inhibition is readily observed at relatively low concentrations $\left(10^{-4}\right.$ $\left.10^{-5} \mathrm{M}\right){ }^{33-34}$ Salicylazosulphapyridine and 5-ASA have been shown to block the lipoxygenase pathway at concentrations found in the stool of patients being treated for ulcerative colitis $(1-10 \mathrm{mM})^{12}$, however, $100 \mu \mathrm{M}$ SASP did not inhibit soybeen lipoxygenase. ${ }^{35}$ In this context, the concentrations used in the present investigation were relevant to analyse.

Another possibility worth raising is that the previously reported lipoxygenase inhibitory effect of SASP $^{12}$ may lead to reduced reactive oxygen species generation, because it has been reported that pro- ducts formed via lipoxygenase may be mediators of $\mathrm{O}_{2}{ }^{-}$generation ${ }^{36}$ and that lipoxygenase pathway may play an important role in $\mathrm{O}_{2}{ }^{-}$production by polymorphonuclear leucocytes. ${ }^{37}$ It is possible that this indirect anti-oxidant effects of SASP and 5-ASA account for some of the anti-inflammatory action of the agents.

These reports and the present findings favour the view that the anti-oxidant action of SASP and/or its metabolites against polymorphonuclear leucocytesderived reactive oxygen species could contribute to its efficacy in ulcerative colitis.

\section{References}

1 Goldman P, Peppercorn MA. Sulfasalazine. $N$ Engl J Med 1975; 293: 20-3.

2 Peppercorn MA, Goldman P. Distribution studies of salicylazosulfapyridine and its metabolites. Gastroenterology 1973; 64: 240-5.

3 Azad Khan AK, Piris J, Truelove SC. An experiment to determine active therapeutic moiety of sulphasalazine. Lancet 1977; 2: 892-5.

4 Klotz U, Maier K, Fischer C, Heinkel K. Therapeutic efficacy of sulfasalazine and its metabolites in patients with ulcerative colitis and Crohn's disease. $N$ Engl J Med 1980; 303: 1499-502.

5 Gould SR. Prostaglandins, ulcerative colitis, and sulphasalazine. Lancet 1975; 2: 988.

6 Sharon P, Ligumsky M, Rachmilewitz D, Zor U. Role of prostaglandins in ulcerative colitis. Gastroenterology 1978; 75: 638-40.

7 Smith PR, Dawson DJ, Swan CH. Prostaglandin synthetase activity in acute ulcerative colitis: effects of treatment with sulphasalazine, codeine phosphate and prednisolone. Gut 1978; 20: 802-5.

8 Harris DW, Smith PR, Swan CH. Determination of prostaglandin synthetase activity in rectal biopsy material and its significance in colonic disease. Gut 1978; 19: $875-7$.

9 Campieri M, Lanfranchi GA, Bazzocchi G, Brignola C, Corazza G, Cortini C, Michelini M, Labo G. Salicylate other than 5-aminosalicylic acid ineffective in ulcerative colitis. Lancet 1978; 2: 993.

10 Gilat T, Ratan J, Rosen P, Peled Y. Prostaglandins and ulcerative colitis. Gastroenterology 1979; 5: 1083.

11 Rampton DS, Sladen GE. Prostaglandin synthesis inhibitors in ulcerative colitis: flurbiprofen compared with conventional treatment. Prostaglandins 1981; 21: 417-25.

12 Stenson WF, Lobos E. Sulfasalazine inhibits the synthesis of chemotactic lipids by neutrophils. J Clin Invest 1982; 69: 494-7.

13 Molin L, Stendahl O. The effect sulfasalazine and its active components on human polymorphonuclear leucocyte function in relation to ulcerative colitis. Acta Med Scand 1979; 206: 451-7.

14 Ōyanagui Y. Inhibition of superoxide anion production in macrophages by anti-inflammatory drugs. Biochem Pharmacol 1976; 25: 1473-80. 
15 Hiller KO, Wilson RL. Hydroxyl-free radicals and antiinflammatory drugs: biological inactivation studies and reaction rate constants. Biochem Pharmacol 1983; 32: 2109-11.

16 Skosey JL, Chow DC, Damgaard E, Sorensen LB. Effects of cytochalasin $B$ on the response of human polymorphonuclear leucocytes to zymosan. J Cell Biol 1981; 57: 237-40.

17 Johnston RB Jr, Lehmeyer JE. Elaboration of toxic oxygen by-products by neutrophils in a model of immune complex disease. J Clin Invest 1976; 57: $836-41$.

18 Root RK, Metcalf JA. $\mathrm{H}_{2} \mathrm{O}_{2}$ release from human granulocytes during phagocytosis. J Clin Invest 1977; 60: 1266-79.

19 Klebanoff SJ, Rosen H. Ethylene formation by polymorphonuclear leukocytes. J Exp Med 1978; 148: 490-506.

20 Allen RC, Loose LD. Phagocytic activation of a luminol-dependent chemiluminescence in rat alveolar and peritoneal macrophages. Biochem Biophys Res Commun 1976; 69: 245-52.

21 McCord JM, Fridovich I. The biology and pathology of oxygen radicals. Ann Intern Med 1978; 89: 122-7.

22 Miyachi Y, Yanase K, Imamura S, Niwa Y. Increased hydroxyl radical generation by normal polymorphonuclear leucocytes incubated in sera from patients with leucocytoclastic vasculitis. Arch Dermatol Res 1982; 274: 65-71.

23 Niwa Y, Miyake S, Sakane T, Shingu M, Yokoyama M. Auto-oxidative damage in Behçet's disease - endothelial cell damage following the elevated oxygen radicals generated by stimulated neutrophils. Clin Exp Immunol 1982; 49: 247-55.

24 Miyachi Y, Niwa Y. Effects of psoriatic sera on the generation of oxygen intermediates by normal polymorphonuclear leukocytes. Arch Dermatol Res 1983; 275: 23-6.

25 Miyachi Y, Imamura S, Niwa Y. Erythema multiforme: A possible pathogenetic role of increased reactive oxygen species. J Clin Lab Immunol 1986; 19: 11-14.

26 Niwa Y, Sakane T, Shingu M, Yanagida I, Komura J, Miyachi $Y$. Neutrophil-generated active oxygens in linear IgA bullous dermatosis. Arch Dermatol 1985; 121: 73-8.

27 Miyachi Y, Uchida K, Komura J, Asada Y, Niwa Y.
Auto-oxidative damage in cement dermatitis. Arch Dermatol Res 1985; 277: 288-92.

28 Niwa Y, Sakane T, Miyachi Y. Dissociation of the inhibitory effect of dapsone on the generation of oxygen intermediates - in comparison with that of colchicine and various scavengers. Biochem Pharmacol 1984; 33: 2355-60.

29 Miyachi Y, Ozaki M, Uchida K, Niwa Y. Effects of thalidomide on the generation of oxygen intermediates by zymosan-stimulated normal polymorphonuclear leukocytes. Arch Dermatol Res 1982; 274: 363-7.

30 Parks DA, Bulkley GB, Granger DN, Hamilton SR, McCord JM. Ischemic injury in the cat small intestine: Role of superoxide radicals. Gastroenterology 1982; 82: 9-15.

31 Lauritsen K, Hansen J, Bytzer P, Bukhave K, RaskMadsen J. Effects of sulphasalazine and disodium azodisalicylate on colonic $\mathrm{PGE}_{2}$ concentrations determined by equilibrium in vivo dialysis of faeces in patients with ulcerative colitis and healthy controls. Gut 1984; 25: $1271-8$.

32 Lauritsen K, Hansen J, Ryde M, Rask-Madsen J. Colonic azodisalicylate metabolism determined by in vivo dialysis in healthy volunteers and patients with ulcerative colitis. Gastroenterology 1984; 86: 1496-500.

33 Hoult JRS, Moore PK. Effects of sulphasalazine and its metabolites on prostaglandin synthesis, inactivation and actions on smooth muscle. Br J Pharmacol 1980; 68: 719-30.

34 Moore PK, Hoult JRS. Selective actions of aspirin- and sulphasalazine-like drugs against prostaglandin synthesis and breakdown. Biochem Pharmacol 1982; 31: 969-71.

35 Marcinkiewicz E, Duniec Z, Robak J. Salazosulfapyridine and non-steroidal antiinflammatory drugs do not inhibit soybeen lipoxygenase. Biochem Pharmacol 1985; 34: 148-9.

36 Smolen JE, Weissmann G. Effects of indomethacin, 5 , 8, 11, 14-eicosatetraynoic acid, and p-bromophenacyl bromide on lysosomal enzyme release and superoxide anion generation by human polymorphonuclear leukocytes. Biochem Pharmacol 1980; 29: 533-8.

37 Yoshimoto S, Yoshimoto T, Tsubura E. Arachidonic acid-induced chemiluminescence of human polymorphonuclear leukoctyes. Biochem Biophys Res Commun 1982; 107: 779-84. 